manner, was distinguished. The tubercular deposits were limited to the upper lobes.

Kidncys.-Both were much enlarged. This was the chief appearance noticed. Possibly, the renal disease may have been an early stage of Bright's disease; or there may have been something like chronic inflammation. There was certainly some slight amount of chronic peri-nephritis present, the capsule adhering firmly to the cortical substance; and it is to be noticed, that there was in the right kidney an appearance of red and white strix, which showed that the cortical substance between the tubuli uriniferi had also been affected with inflammation, a morbid appearance described by Rayer. The alkaline urine, taken in connexion with these appearances, may thus have been due, contrary to the opinion given, to this inflammation. We have also proof that the renal secretion was imperfect, first-in the general liquidity of the blood after death, and rapid decomposition of the body, as is commonly observed in cases where there has been a morbid quantity of urea circulating in it; and secondly-we have the positive evidence of the presence of urea in the fact that it was detected in the serum effused in the brain.

Causes.-1. Of the renal disease. There does not appear to have been any very evident predisposing cause. Dissipated habits formed the only apparent exciting cause.

2. Of the cerebral discase.-Among the predisposing we may enumerate, first, his scrofulous diathesis; secondly, his sex; cerebral disease being gencrally more common in inen, (Parent and Martinet;) thirdly, his studious habits.

The exciting cause appears to have been the renal disease. The presence of a morbid quantity of urea in the blood, we know does give rise to intercurrent inflammations in the varions organs of the body.

The prognosis from the first was unfavourable. As the disease frogressed, its obstinacy under treatment and his ex treme weakness saemed to point but too distinctly to a fatal termination.

\section{ROYAL SOUTH LONDON DISPENSARY.}

\section{EMPYEMA AND PNEUMOTIORAX. \\ Reported by WILLIAM TIFFIN ILIFF, Jcx., Esq., SURGEON TO THR DISPENSARY.}

THe case here related is one of empyema, or chronic pleuritic effusion, and will be found, $I$ think, to present numerous points of interest; and as the subject of the propriety of performing the operation of paracentesis thoracis is still a vexata quasitio, it is the duty of every member of the profession to contribute, fairly and impartially, whatever cases may occur to him bearing on any important point, as in this way alone can we arrive at the true value of any mode of treatment.

Samuel M - aged thirty-rive, was admitted under my care as out-patient at the dispensary, February 13th, 1846, complaining of cough, with seanty expectoration, oppression of breathing, occasional gnawing pain in the left side, and inability to lie on the right. He is a Scotchman, of spare habit, by occupation a traveller; has drunk freely at times, but not to excuss; states that his health has been, on the whole, goud, until nive weeks ago, when ho had a serere attack of inflammation of the le't side, for which he was leeched, blistered, \&c., and from the effects of which attack he is now suffering. A very cursory examination of his chest showed resonance of the rigit side on percussion, and universal dulness of the left, except at theapex. Ordered, mucilage mixture, an ounce; dilute sulphuric acid, eight minims; ipecacuanha wine, fifteen minims. Mix, and take three times a day. Compound squill pill, four grains, with blue pill, one grain; night and morning. Feeling a little relieved by this, he shortly ceased to attend.

March 7 th.-I was sent for to see him at his honse. Fis symptoms were as before, but increased in degree. No fixed pain, but general oppression of breathing, especially at night, with congh, viseid, clear expectoration, and occasional specks of blood, most probably from the fauces; ribs imperfectly raised in inspiration; right side resonant, excent in the inframammary region, where the heart is situated; respiration puerile; left sirle unirersally duil ; very faint and distant respiratory murmur anteriorly, with loss of voice; posteriorly, tubular respiration with brunchophony, especially audible near the spine; no material difference detected by measurement; sunds of the heart normal, its position to the right of the sternum; tongue white; bowels costive; skin perspirable; pulse $3 \mathrm{~J}$, soft, and rather woak. Ordered, a large blister to the side. Sulphate of magnesia, two drachms; carbonate of magnesia, half a scruple; peppermint water, an ounce; direetly.
Repeat the pill three times a day. Tincture of hemlock, half a drachm; wine of ipecacuania, fifteen minims; water, an ounce. Mix, and take three times a day.

17th.-Symptoms more urgent; he complains of cold chills in the afternoon; paroxysms of congh and dyspnoea very severe, especially towards night; tongue clean; bowels regular; urine containing slight flocculent matter, specifie gravity 1016 , temp. $50^{\circ}$, depositing phosphates by heat, though not copiously; no trace of albumen. Careful measurement of the entire chest, just below the nipple, thirty-nine inches and threequarters, right side nineteen inches and a half, left, twenty inches and a quarter; apparently, more rotundity of the left side, and tenderness in the intercostal spaces posteriorly and inferiorly; respiration twenty-six in a minute ; no enlargement of the liver. His treatment since last report has been, free blistering, the squill and blue pill, and ralious tonic expectorant mixtures. Believing this to be a case of chronic pleuritic effusion, and knowing the interest Dr. IIughes took in these cases, I requested his opinion, with a view to the removal of the fluid; and as he advanced no objection, I introduced the exploring trocar between the ninth and tenth ribs, nearly six inches from the spine, and on fluid flowing in a full stream, I withdrew the camula, and introducing a larger one (one-twelfth of an inch in diameter) drew off nine pints and three-quarters. It was not until quite the conclusion, that any jerk was manifested in the flow of the fluid. No air entered, and the wound was closed with lint and strapping. He bore the operation very well, and suffered no distress until towarrs its conclusion, when he felt somewhat faint, and slight congh arose. On being laid comfortably in bed, the pulse was $100^{\circ}$, and better than before the operation; respiration thirty, unaccompanied by pain; less oppression of breathing; percussion over the left side, anteriorly, elicited great resonance. Ordered, Battley's sedative solution, fifteen minims; solution of acetate of ammonia, two drachms; camphor mixture, an ounce; directly.-Nine P.M.: Very comfortable; expresses himself as greatly relieved; respiration thirty; pulse 96 , soft ; skin perspirable; he has dozed a little. Ordered, blue pill, five grains, at night; and next morning, arrowroot, \&c.; and a draught at bedtime, containing Battley's solution, seven minims. The fluid withdrawn was of a reddish colour, very sticky, specific gravity $1025-52^{\circ}$, moderately alkaline, of faint odour, almost entirely converted into coagulum by heat. By standing, about four ounces of purnlent matter were deposited.

1sth.-Very comfortable; has had a good night. Blue pill, four grains; powdered opium, one sixth of a grain, night and morning. Compound infusion of gentian, an ounce; iodine, one-eighth of a grain; hydriodate of potash, five grains; wine of ipecacuanha, twenty minims, three times a day.

19th.-Occasional cough, but much less severe; no increase of respiratory sounds. Free blistering to the side.

21st.- He lay on his right side some little time last night, and to-day, withont inconvenience; cough a little increased again ; respiration, 24 ; pulse 108 , weak ; tongue white, slightly furred. To have meat, and half a pint of mild ale, daily.

24 th.- Much the same. On the $22 \mathrm{nd}$, he had a severe attack of colic, inducing faintness and general pallor, but subsiding on free evacuation of his bowels. Ordered yesterday, for mixture, infusion of gentian, with dilute nitric acid. Respira. tion 26 ; yulse 100 ; sleep good; bowels remular; appetite improving. He was up yesterday evening without inconvenience. ixamination of his chest elicits, anteriorly, general and complete preternatural resonance of the left side, except at the lower part, as also laterally; this latter was removed by turning him suddenly on his right side, a splashing sound being audible. Posteriorly, dulness over the lower half; $a b$. normal resonance over the upper; respiratory sounds nearly inaudible, except at the apex; distinct ægophonic bronchophony superiorly and posteriorly; no sound conveyed to the applied ear on coughing.

April 6 th.-The sound on succussion having excited my attention, I inquired particularly as to its previous existence, when he informed me that he first heard it when recovering from the acute attack before I saw him, when he mentioned it to his medical attendant, and that it was heard by one or two persons in the house. He also said, that he had for three years been the subjeet of slight cough and cold, but no pain inside, no drspncea, no sweats, nor imalility to lie on either side. Wis pulsu is now 100, soft, and weak; bowels regular; mouth not affected; sleep good; is perhaps losing flesh; cungh much the same; not worse at any particular time, except for the last week, when lying on the right side; in the recumbent position the splashing sound is very clear on succussion; resonance on boilh sides, superiorly and anteriorly, to about an iach below 
the mamme; the dulness on the right cansed by the heart does not extend into the axilla; on the left it does, and is now unaffected by his lying on the right side. Posteriorly, (position erect,) resonance on the right; dulness on the left. Anteriorly and superiorly on the left side, stridulous respiratory sounds; inferiorly, faint and distant tubular respiration most audible between the sternum and left mamma; entire absence of voice, except at the apex. Posteriorly, respiratory murmur in the supra-scapular region decreasing in intensity inferiorly, but not entirely lost. Right side as before; the sounds of the heart most audible beneath the right nipple, but also audible as far as the left axilla. Continue the medicines; the compound iodine ointment to be applied to the blistered surface. Wine, four ounces, arrowroot, and meat, or broth, daily.

28th.-The paroxysms of cough have returned with severe retehing, and are present night and morning; auscultation has shown great increase of the fluid, without any expansion of the lung; the splashing sound is still andible; in the supine position there is resonance below the clavicle, but dulness on his assuming the erect position, [the former probably owing to the presence of a thin stratum of air between the fluid and parietes ?] posteriorly, the dulness is general over the left side. His treatment has consisted of the squill and blue pill, tonics and expectorants, and the application of compound iodine ointment, three parts, with strong mercurial ointment, one part to the blistered surface. On the $24 \mathrm{th}$, I measured his chest--each side about eighteen inches and three quarters. Urine, on that day, $1010-60^{\circ}$, alkaline, phosphatic not albuminous. I again tapped his chest, and drew off rather more than four pints, introducing the trocar one space higher. The intercostal spaces were widened, and more tense. The fluid did not flow so equably, but more in a jerk, and synchronous with respiration; two or three bubbles (certainly not more) of air entered the chest just at the conclusion. He bore the operation well. Battley's sedative solution, fifteen minims, directly, as before.-Nine P.M.: Lying easily on his right side; pulse rallying. He is very comfortable, and has not had his evening paroxysm. The fluid was of a dirty greenish-yellow colour, turbid, nearly inodorous, slightly alkaline, specific gravity $1025.66^{\circ}$; by heat converted into a solid mass. Three ounces, by standing twenty-four hours, separated into equal parts of clear fluid and purulent sediment.

May 23rd. $\rightarrow$ Since last report little new has occurred, the fluid has rapidly re-accumulated, and the cough and retching are returned, chiefly evening and morning, and lasting about half an hour; expectoration purely bronchitic; he has also suffered from sore-throat, laryngeal irritation, husky voice, and feeling of dryness in the fauces; respiration $2 \tau$; pulse 110 , soft, full, but weak; the heart, which at one time had advanced considerably towards its normal position, has again resumed its situation beneath the right mamma; dulness nearly complete over the left side, the splashing sound faint; intercostal spaces widened and tense; fluctuation felt very clearly on coughing. I first observed, a few weeks ago, that the left nipple is on a plane higher than the right. He has been out walking; has continued his wine, and enjoys his nourishment. The same treatment has been persisted in, with the addition of the dilute hydrocyanic acid, with the hope of relieving the retching and cough.

25th.-I was called to him at five P.M.; he had just been at tacked with a kind of nervous delirium, and would have fallen had he not been supported; direct cause unknown, as he was merely walking from one room to another. On my arrival he evinced great fright and childishness. Pulse 120 , weak; no increase of dyspnoa; pupils natural; bowels regular; free perspiration. It appeared, about a fortnight previously, he had been much frightened on suddenly turning and seeing limself in a looking-glass which he thought was in another part of the room. To be kept quiet; omit his medicines. Battley's sedative solution, five minims, in camphor mixture, directly.-Nine P.M.: Summoned again; I found him in a pro* fuse clammy sweat; at one time nearly fainting, at another, shivering intensely; countenance anxious; pulse 130, weak; very restless, with great depression of spirits; rational, however; no increase of dyspnoea, \&c. Ordered, wine and arrowroot. Ammonia, and spirit of lavender, in camphor mixture, frequently.

26th.- Very comfortable; no return of the shivering or nervous state since I saw him; has had a very good night; no increase of congh, rather the contrary; bowels open; pulse 108, rallying. To resume his pill night and morning. Compound infusion of gentian, an ounce; aromatic spirit of ammonia, half a drachm; tincture of henbane, fifteen minims; tincture of ginger, ten minims: mix, and take three times a day. Wine of opium, five minims; camphor mixture, an ounce every night.

29th.-Ten A.M.: Yesterday evening, on getting into bed, delirium returned; he has been at times violent, but generally more restless; in some degree sensible. I found him quiet, lying in a crouching attitude; mind wandering; pulse 130, very weak; pupils equal, sluggish, slightly dilated; urine free; breathing apparently more easy. Cold to the head. Omit the medicines. Wine and nourishment occasionally.-Three P.M.: Has been quiet; skin hot, almost pneumonic, and inclined to dry; pulse 125; half conscious, but muttering continually "aganiskan;" breathing 27; tongue coated and white, not voluntarily protruded; bowels not opened since the 27 th. The head to be shared, and vinegar and water applied. Omit the wine. Salts and magnesia, an ounce directly.--Eight P.M.: Has been rather better, and spoken a few words; respiration 30 , rather stertorous; position on the left side; respiratory murmur on the right loud and cooing; pulse 120; skin warm, not perspirable; bowels not yet open. Protochloride of mercury, five grains directly; magnesia and salts an ounce and a half, in half an hour after. Protochloride of mercury, compound antimonial powder, of each two grains every three hours.

30th.- Half-past nine A.M.: About three hours since, a severe attack of coughing roused him, and he has been quite sensible ever since, and can speak as usual. Bowels not yet open, skin perspirable; pupils natural; pain at the top of the head; pulse 100, feeble; position, on the right side. Ordered, magnesia and salts, an onnce and a half every three hours, until the bowels are opened; after which, an ounce to be taken twice a day. Continue the powder night and morning. Broth and arrow-root.

June 1st.-Bowels freely opened, but not purged; pain in head less; position variable; no increase of cough; expectoration, as before, viscid, clear, and a little frothy; stomach irritable; urine of an orange-yellow colour, faint odour, slightly alkaline and phosphatic, no albumen, $1009-70^{\circ}$ Ordered, powdered rhubarb, four grains; mercury with chalk, one grain, night and morning; effervescing draught, with infusion of gentian, every six hours.

2nd.-Soon after I left, he became much worse, nervous, delirious, and restless, with failing consciousness; does not use the left arm and leg much; the right arm he does very much; no decided paralysis of the former, however: pupils equal, rather staring.

3rd.-Six A.x.: He died, no new symptom having occurred.

Necropsy, twenty-nine hours after death.-Weather intensely hot. Thin, but not extreme emaciation; aspect calm, slightly smiling; discoloration commencing about abdomen and back. On removing the sternum and cartilages of the ribs, to which the left pleura strongly adhered, the heart was observed toward the right side; the left pleura contained nearly eleven pints of almost inodorous, sero-purulent fluid. The pleura was covered, or rather lined throughout, with a thick, firm, almost cartilaginous deposit, and was narrowed somewhat at the upper part, so as to form a kind of sacculated apex.-Thoracic viscera removed entire: a tube was passed into the left bronchus, and inflation attempted; no air could be found to escape. The lung, which had been very much compressed against the spine, was dark, dense, and fleshy; quite impermeable to air; slight tubercular deposit. Right lung was adherent, at the apex, by slight yet strong bands; presented tubercles irregularly scattered through the apex chiefly, where was one very small cavity in the substance of the lung. In the base of the lung, there were but few tubercles.-Heart, large and flabby; no disease of the valves, or deposit on the aorta.-Blood, very dark, and on the whole, fluid.-Liver, granular, whitey-brown in colour.-Spleen, healthy.-Kidneys, large, flabby, rather dark. - The hecd was not examined.

The remarks on this case must be postponed to a future number.

Marriages.-More marriages were celebrated in 1844 than had ever before been registered in England. The number was 132,249 . In 1843 , the number was 123,818 , more by 4993 than were registered in 1842 . The 118,825 marriages in 1842 were much below the average number; so few had not taken place in any year since 1832. The proportion of marriages to the population went on declining from 1839 to 1812 , increased in 1843, and attained the maximum in 1841. The fluctuations were greatest in the North-western Division, Yorkshire, and the metropolis. In the Eastern and Southern Divisions the changes were not considerable.-Seventh A nmul Report of the Registrar-General, 1846. 